\title{
Construction of a lentiviral vector containing shRNA targeting ADAM17 and its role in attenuating endotoxemia in mice
}

\author{
BING HE ${ }^{*}$, XIAOOU LI*, TUO HU, WENJING LIAN and MINGXIA ZHANG \\ Department of Pediatrics, Renming Hospital, Wuhan University, Wuhan, Hubei 430060, P.R. China
}

Received October 10, 2016; Accepted June 26, 2017

DOI: $10.3892 / \mathrm{mmr} .2017 .7307$

\begin{abstract}
Systemic inflammatory response syndrome is a pathophysiological inflammatory response mediated largely by tumor necrosis factor- $\alpha$ (TNF- $\alpha)$, in response to infectious or non-infectious stimuli. TNF- $\alpha$ secretion in response to bacterial lipopolysaccharide (LPS) is regulated in part by disintegrin and metalloproteinase domain-containing protein 17 (ADAM17). Therefore, the present study aimed to identify an effective inhibitor of ADAM17, in order to control inflammation and associated processes. In the present study, a lentiviral vector expressing short hairpin (sh)RNA targeting the ADAM17 gene was constructed. U937 cells were infected with the lentivirus and stimulated with LPS. ADAM17 expression was assessed by western blotting and TNF- $\alpha$ secretion was assessed by ELISA analysis. The lentivirus was additionally tested in vivo in a mouse model of endotoxemia and sTNF- $\alpha$ expression was assessed by flow cytometry in peritoneal macrophages. In vitro, the ADAM17 shRNA lentivirus reduced ADAM17 expression, and prevented TNF- $\alpha$ maturation in U937 cells. In vivo, mice exposed to the ADAM17 shRNA lentivirus prior to LPS-induced endotoxemia exhibited fewer signs of inflammation and less tissue damage compared with the control mice. In conclusion, the present study successfully constructed a shRNA lentiviral vector targeting the ADAM17 gene that exhibited apparent in vitro and in vivo effects on TNF- $\alpha$ processing in response to an LPS challenge. The results of the present study may aid the
\end{abstract}

Correspondence to: Dr Bing He, Department of Pediatrics, Renming Hospital, Wuhan University, 99 Zhang Zhi Dong Street, Wuchang, Wuhan, Hubei 430060, P.R. China

E-mail: hb0701@sina.com

*Contributed equally

Abbreviations: SIRS, systemic inflammatory response syndrome; TNF- $\alpha$, tumor necrosis factor- $\alpha$; LPS, lipopolysaccharide; MOF, multiple organ failure; mTNF- $\alpha$, transmembrane TNF- $\alpha$; sTNF- $\alpha$, secreted TNF- $\alpha$; ADAM, disintegrin and metalloproteinase domain-containing protein; TACE, TNF- $\alpha$ converting enzyme

Key words: disintegrin and metalloproteinase domain-containing protein 17 , lentivirus, short hairpin RNA, endotoxemia design and improvement of drugs designed to inhibit the function of ADAM17, and suggested a novel means of controlling inflammation and associated processes.

\section{Introduction}

Systemic inflammatory response syndrome (SIRS) is caused by an inappropriately strong inflammatory reaction to infectious or non-infectious stimuli, and results in the release of humoral and cellular inflammatory mediators (1). While a certain degree of inflammation is appropriate and protective, SIRS is a pathophysiological process that may cause extensive tissue injury (2). SIRS has become a leading cause of mortality in patients with infectious disease (3). SIRS occurs as an intermediate step in a pathophysiological process that spans: i) Injury; ii) stress responses; iii) SIRS with multiple organ dysfunction syndrome; and eventually iv) multiple organ failure (MOF) (4). Notably, although SIRS is a severe complication, there is the opportunity for a cure or reversal of the pathophysiological process prior to MOF.

Infections are an important cause of SIRS, and $>80 \%$ of bacterial infections are caused by Gram-negative bacteria that produce lipopolysaccharide (LPS), which is one of the principal triggers of SIRS $(5,6)$. Inflammatory cells, including activated neutrophils and granulocytes, may exert an important role in the pathophysiological processes of $\operatorname{SIRS}(7,8)$. In addition, tumor necrosis factor- $\alpha(\mathrm{TNF}-\alpha)$ is an important inflammatory mediator released from cells stimulated by LPS (9). TNF- $\alpha$ is additionally hypothesized to be an important contributor to SIRS, as it appears early, peaks rapidly and exhibits numerous host functions (10). TNF- $\alpha$ exists as a transmembrane protein (mTNF- $\alpha ; 26 \mathrm{kDa})$ that is cleaved by a metalloproteinase to release the ectodomain, which becomes mature secreted TNF- $\alpha$ (sTNF- $\alpha ; 17 \mathrm{kDa})$ during the inflammatory response (11). High levels of sTNF- $\alpha$ are cytotoxic and a major contributor to inflammation-mediated tissue injury (12). Therefore, a better understanding of how mature TNF- $\alpha$ is produced, and its biological function, may inform the development of novel therapeutic strategies to effectively inhibit LPS-induced TNF- $\alpha$ overproduction, thereby preventing SIRS and MOF.

mTNF- $\alpha$ is processed by either matrix metalloproteinases or disintegrin and metalloproteinase domain-containing proteins (ADAMs). ADAM17, also termed TNF- $\alpha$ converting enzyme (TACE), is highly specific for mTNF- $\alpha$ and cleaves it 
to produce sTNF- $\alpha$ by hydrolyzing mTNF- $\alpha$ between Ala76 and Vla77 (12-14). Therefore, a potential method for controlling the toxicity of $\mathrm{sTNF}-\alpha$ is to develop TACE-specific inhibitors.

The present study aimed to develop a novel platform for inhibiting the LPS-induced inflammatory reaction leading to SIRS. Therefore, a recombinant lentiviral vector was developed to deliver short hairpin (sh)RNA targeting ADAM17, to effectively inhibit ADAM17 expression. The vector was tested in vitro using U937 cells stimulated with LPS, and in vivo in a mouse model of endotoxemia.

\section{Materials and methods}

Ethics statement. The present study was performed in strict accordance with the recommendations in the Guide for the Care and Use of Laboratory Animals (15) of the National Institutes of Health (Bethesda, MD, USA). The protocol was approved by the Committee on the Ethics of Animal Experiments of Wuhan University (Wuhan, China; permit no. WDRY2015-K006). All surgery was performed under $30 \mathrm{mg} / \mathrm{kg}$ sodium pentobarbital anesthesia, and all efforts were made to minimize suffering.

Reagents. T4 DNA ligase and the Plasmid Maxi kit were purchased from Qiagen China Co., Ltd. (Shanghai, China). Human cell lines 293 T and U937 were purchased from the Experimental Cell Center of Wuhan University, and Escherichia coli DH5 $\alpha$ were maintained in the laboratory. Lentiviral vectors and primers were synthesized by Shanghai GeneChem Co., Ltd. (Shanghai, China). Kunming mice were purchased from the Laboratory Animal Center, Tongji Medical College (Wuhan, China). Other reagents noted below were analytical grade (Takara Biotechnology Co., Ltd., Dalian, China). The U937 cell line was authenticated by short tandem repeat profiling conducted by Shanghai Zhong Qiao Xin Zhou Biotechnology Co., Ltd. (Shanghai, China; sample no. 20170303-20).

Construction of lentiviral vectors expressing shRNA. The shRNA sequence used to target ADAM17 was the same as that previously described (11). Synthetic oligonucleotide sequences were constructed, and annealed to create double-stranded DNA using the following sequences: forward, 5'-ccggcc TGG TTACAACTCATGAATTctcgagAATTCATGAGTTGATTG TAACCAggtttttg-3' and reverse, 5'-aattcaaaaaccTGGTTA CATGAATTctcgagAATTCATGAGTTGTAACCAgg-3'. The sequence written in uppercase represents the stem and lowercase sequences are the loop. The target gene was inserted into the AgeI and EcoRI cleaved pGC-LV vector by homologous recombination using the T4 DNA ligase enzyme. Competent DH5 $\alpha$ cells were prepared with calcium chloride and subsequently transformed. Positive clones were selected by polymerase chain reaction (PCR) analysis using Taq enzyme (cat. no. DR010s; Takara Biotechnology Co., Ltd.). The following primer sequences were used for PCR: Forward, 5'-ccatgattccttcatatttgc-3' and reverse, 5'-cgcgtggataaccgtattac-3'. The thermocycling conditions for PCR were as follows: $5 \mathrm{~min}$ at $94^{\circ} \mathrm{C}$, followed by 30 cycles of $30 \mathrm{sec}$ at $94^{\circ} \mathrm{C}, 30 \mathrm{sec}$ at $60^{\circ} \mathrm{C}$ and $30 \mathrm{sec}$ at $72^{\circ} \mathrm{C}$, and a final extension at $72^{\circ} \mathrm{C}$ for $6 \mathrm{~min}$. The recombinant positive clones were sent to Invitrogen (Thermo Fisher Scientific, Inc., Waltham, MA, USA) for sequencing.

Production of the lentiviral vector. 293T cells in the logarithmic growth phase were digested with trypsin $24 \mathrm{~h}$ prior to being transfected. The cells were resuspended at $6.0 \times 10^{8}$ cells/ 1 in Dulbecco's modified Eagle's medium containing $10 \%$ fetal bovine serum (FBS) and maintained at $37^{\circ} \mathrm{C}$ in an atmosphere containing $5 \% \mathrm{CO}_{2}$ for $24 \mathrm{~h}$ until the cells were $50-60 \%$ confluent. The cell culture medium was replaced with serum-free medium $2 \mathrm{~h}$ prior to transfection. DNA was extracted using the Plasmid Maxi kit (Qiagen China Co., Ltd.), according to the manufacturer's instructions. A solution containing the prepared DNA (20 $\mu \mathrm{g}$ pGC-LV-shRNA vector, $15 \mu \mathrm{g}$ pHelper vector 1.0, and $10 \mu \mathrm{g}$ pHelper vector 2.0) was added to a sterile centrifuge tube and the final volume was brought to $2.5 \mathrm{ml}$. The solution was incubated at room temperature for $5 \mathrm{~min}$, and $293 \mathrm{~T}$ cells were transfected using Lipofectamine ${ }^{\mathrm{TM}} 2000$ (Invitrogen; Thermo Fisher Scientific, Inc.) according to the manufacturer's instruction. The transfected cells were incubated in serum-free medium for $8 \mathrm{~h}$, the medium was subsequently replaced with medium containing $10 \%$ serum, and the cells were cultured for an additional $48-72 \mathrm{~h}$.

The culture supernatants were collected when the cells expressed high levels of green fluorescence and cell fusion was observed (48-72 h). The virus in the supernatant was concentrated to the target volume by centrifugation $\left(4^{\circ} \mathrm{C}, 4,000 \mathrm{x} \mathrm{g}\right.$, $10 \mathrm{~min})$. The virus concentrate was removed and aliquoted into a virus tube for long-term storage at $-80^{\circ} \mathrm{C}$. In order to determine the viral titer, $100 \mu \mathrm{l}$ viral stock was used to make $1 \mathrm{ml}$ serial dilutions $\left(10^{-2}-10^{-6}\right)$, which were used to infect $293 \mathrm{~T}$ cells in a six-well plate ( $2 \times 10^{5}$ cells). Following $48 \mathrm{~h}$ in culture, green fluorescent protein (GFP) expression in each well was observed under a fluorescence microscope (magnification, $\mathrm{x} 200$ ). The number of cells expressing GFP was counted and multiplied by the dilution ratio to determine the viral titer [transducing units (TU)/ml].

Assessing the cytotoxicity of the lentiviral vector. U937 cells were grown in RPMI-1640 medium (Sigma-Aldrich; Merck KGaA, Darmstadt, Germany) containing 10\% FBS. When the cells had reached $10^{5}-10^{6}$ cells $/ \mathrm{ml}$, they were diluted to $5 \times 10^{4}$ cells $/ \mathrm{ml}$ with RMPI-1640, inoculated into a 6 -well plate and maintained in $5 \% \mathrm{CO}_{2}$ at $37^{\circ} \mathrm{C}$ for $12 \mathrm{~h}$. Recombinant lentivirus $(0.5 \mathrm{ml}, 100 \mathrm{ng} / \mathrm{ml})$ was added and the empty vector was used as a control. After $12 \mathrm{~h}$, the condition of the cells was observed. If there was no evidence of a significant cytotoxic effect, the culture was continued for $24 \mathrm{~h}$ prior to changing the culture medium. If cytotoxic effects were observed, the medium was changed immediately. The GFP expression was observed 3 days subsequent to infection, using a fluorescence microscope.

Detection of protein expression by western blotting. Total proteins from U937 cells infected with the ADAM17 shRNA lentiviral vector or a negative control were extracted using the radioimmunoprecipitation assay (RIPA; 1\% Triton-100, $50 \mathrm{mmol} / 1 \mathrm{TrisHCl}, 150 \mathrm{mmol} / 1 \mathrm{NaCl}, 0.1 \mathrm{mmol} / 1$ phenylmethylsulfonyl fluoride, $1 \mu \mathrm{mol} / 1$ pepstatin, $0.5 \mathrm{mg} / \mathrm{ml}$ leupeptin and $0.3 \mu \mathrm{mol} / 1$ aprotinin) method, and protein concentrations 
were measured using a bicinchoninic acid assay. LPS (Sigma-Aldrich; Merck KGaA) stimulation with $2.5 \mathrm{mg} / \mathrm{l}$ LPS was performed prior to western blotting for $6 \mathrm{~h}$ at room temperature. Total protein $(50 \mu \mathrm{g})$ was separated by $10 \%$ SDS-PAGE at $300 \mathrm{~mA}$ for $2 \mathrm{~h}$, followed by transfer to a nitrocellulose membrane. The membrane was blocked for $2 \mathrm{~h}$ in TBS-Tween-20 (20\% Tween-20; TBST) and 5\% nonfat dry milk at room temperature, and subsequently incubated with a primary antibody against human ADAM17 (cat. no. 14-6202; 1:600; Abcam, Cambridge, UK) at $4^{\circ} \mathrm{C}$ overnight. Subsequently, the membrane was washed three times with TBST, and incubated with a horseradish peroxidase-labeled secondary antibody (cat. no. sc-2004; 1:1,000; Santa Cruz Biotechnology, Inc., Dallas, TX, USA) at room temperature for $1 \mathrm{~h}$. The membrane was washed three times with TBST and the bands were visualized using enhanced chemiluminescence (PerkinElmer, Inc., Waltham, MA, USA). The film was scanned or photographed, and analyzed using a gel imaging processing system (Beijing Sage Creation Science Co., Ltd., Beijing, China; GSD800 system). The results were analyzed using Quantity One software v4.62 (Bio-Rad Laboratories, Inc., Hercules, CA, USA). $\beta$-actin (1:600; cat. no. sc-69879; Santa Cruz Biotechnology, Inc.) was used as the internal control. The experiment was repeated three times.

Measuring sTNF- $\alpha$ from U937 cells following LPS stimulation using ELISA analysis. A total of three groups underwent the following experiment: The shRNA + LPS group, the LPS + blank vector group, and a blank vector without LPS stimulation as the control group. In the shRNA + LPS group, the cells $\left(1 \times 10^{5} / \mathrm{ml}\right.$; in a 12 -well plate at $1 \mathrm{ml} /$ well $)$ were infected with the ADAM17 shRNA vector for $66 \mathrm{~h}$ and were subsequently stimulated with $2.5 \mathrm{mg} / \mathrm{l}$ LPS (12) for an additional $6 \mathrm{~h}$ at room temperature. The LPS group was stimulated with $2.5 \mathrm{mg} / \mathrm{l} \mathrm{LPS}$ for $6 \mathrm{~h}$ at room temperature. The supernatants from each well were collected and the sTNF- $\alpha$ levels were measured using an ELISA performed according to the manufacturer's protocol (cat. no. 550610; BD Biosciences, Franklin Lakes, NJ, USA). Using a microplate reader, the absorbance of each well was detected at $495 \mathrm{~nm}$.

Measuring mTNF- $\alpha$ on the surface of U937 cells following LPS stimulation using western blotting. Western blotting was used to detect the expression of mTNF- $\alpha$ on the surface of the U937 cells in the shRNA + LPS, LPS and control groups. The RIPA method was used to extract the mTNF- $\alpha$ proteins from U937 cells. Proteins were probed with the rabbit anti-human mTNF- $\alpha$ primary antibody (cat. no. ab169616; 1:600; Abcam). The detailed experimental procedure was the same as for the aforementioned detection of ADAM17 protein.

Assessing the in vivo effects of ADAM17 knockdown. Specific pathogen-free grade Kunming mice (male; $n=18 ; \sim 20$ g; 8 weeks old) were randomly divided into three groups ( $n=6 /$ group). Rats were housed 2 or 3 to a cage under specific pathogen-free conditions (controlled temperature of $24 \pm 3^{\circ} \mathrm{C}$ and humidity of $55 \pm 15 \%$ ) with a $12-\mathrm{h}$ light/dark cycle and ad libitum access to tap water and food. The three treatment groups were: i) PBS control group; ii) endotoxemia group; iii) endotoxemia + lentivirus group. Endotoxemia was established by injecting $0.1 \mathrm{ml}$ of a solution containing $10 \mathrm{mg} \mathrm{D}$-ammonium galactosamine and $2 \mu \mathrm{g}$ LPS into the caudal vein (16). For the endotoxemia + lentivirus group, $48 \mathrm{~h}$ prior to inducing endotoxemia, the mice were injected with the shRNA ADAM17 lentivirus ( $4 \times 10^{8} \mathrm{TU} /$ mouse) through the caudal vein. The mice in the control group were injected with the blank vector in the same manner as those in the endotoxemia + lentivirus group. The reaction of each group of mice was observed. The mice were sacrificed $6 \mathrm{~h}$ post-LPS injection using cervical dislocation. Sterile saline $(5 \mathrm{ml})$ was immediately injected into the abdomen, which was gently massaged for $1 \mathrm{~min}$. Subsequently, $10 \mathrm{ml}$ peritoneal fluid was extracted into a centrifuge tube and centrifuged for $5 \mathrm{~min}$ at $1,000 \mathrm{x} \mathrm{g}$ at room temperature. The cells were washed twice with PBS, resuspended in RPMI-1640 containing $10 \% \mathrm{FBS}$ and cultured at $37^{\circ} \mathrm{C}$ under $5 \% \mathrm{CO}_{2}$ for $1 \mathrm{~h}$. Once the cells had adhered to the wall of the tube, the non-adherent cells were removed and fresh medium was added.

The livers, kidneys and lungs were also removed. Some tissues were used immediately to prepare frozen sections (at $-4^{\circ} \mathrm{C} ; 7 \mathrm{~mm}$ sections) for the detection of GFP expression under a fluorescence microscope (BX53; Olympus Corporation, Tokyo, Japan). The remaining tissues were collected and fixed with $4 \%$ paraformaldehyde for $24 \mathrm{~h}$ at room temperature then dehydrated $(75 \%$ alcohol for 4 h, $85 \%$ alcohol for 2 h, $90 \%$ alcohol for $1.5 \mathrm{~h}, 95 \%$ alcohol for $1 \mathrm{~h}$, absolute ethanol I for $30 \mathrm{~min}$ and absolute ethanol II for $30 \mathrm{~min}$ ), cleared, dipped in wax $\left(60^{\circ} \mathrm{C}\right.$ for $1 \mathrm{~h}$, three times), paraffin-embedded and sliced into sections (4-mm). Following dewaxing (xylene I for $10 \mathrm{~min}$, xylene II for $10 \mathrm{~min}$, absolute ethanol I for $5 \mathrm{~min}$, absolute ethanol for $5 \mathrm{~min}, 95 \%$ alcohol for $3 \mathrm{~min}, 85 \%$ alcohol for $2 \mathrm{~min}, 70 \%$ alcohol for $2 \mathrm{~min}$ and distilled water for $2 \mathrm{~min}$ ), the sections were stained with hematoxylin for $7 \mathrm{~min}$ at room temperature, and then counterstained with eosin for $2 \mathrm{~min}$ at room temperature. The stained tissues were observed under a fluorescence microscope (BX53; Olympus Corporation) and images were captured for analysis; a total of 10 fields of view were observed.

Flow cytometric analysis of mTNF- $\alpha$ on the surface of peritoneal macrophages. Following trypsinization and two washes with PBS containing 5\% bovine serum albumin (BSA; Sigma-Aldrich; Merck KGaA), the murine peritoneal macrophages were resuspended at $10^{6}$ cells $/ \mathrm{ml}(1 \mathrm{ml})$. Following blocking with PBS containing 5\% BSA (Sigma-Aldrich; Merck $\mathrm{KGaA}$ ) for $1 \mathrm{~h}$ at $4^{\circ} \mathrm{C}$, the cells were incubated with a primary goat anti-mouse TNF- $\alpha$ polyclonal antibody (cat. no. ab8348; 1:100; Abcam) for $1 \mathrm{~h}$ at $4^{\circ} \mathrm{C}$. The cells were washed twice with PBS, followed by incubation with a FITC-conjugated rabbit anti-goat secondary antibody (cat. no. BA1101; 1:60; Boster Biological Technology, Pleasanton, CA, USA) for $1 \mathrm{~h}$ at $4^{\circ} \mathrm{C}$ in the dark. Following centrifugation $400 \mathrm{x}$ g for $2 \mathrm{~min}$ at $4^{\circ} \mathrm{C}$ ) and two washes with PBS, the cells were resuspended in PBS at $4^{\circ} \mathrm{C}$ and immediately subjected to flow cytometry (BD FACSCalibur ${ }^{\mathrm{TM}}$ flow cytometer and analysis using BD CellQuest Pro software version 5.2.1 (both BD Biosciences). The average fluorescence of $5 \times 10^{4}$ cells was used to determine the expression levels of mTNF- $\alpha$.

Statistical analysis. Experiments were repeated three times and statistical analyses were performed using SPSS version 
Table I. sTNF- $\alpha$ concentration in U937 culture supernatants (mean \pm standard deviation; $\mathrm{n}=3$ ).

\begin{tabular}{lc}
\hline Group & Concentration of sTNF- $\alpha, \mu \mathrm{g} / 1$ \\
\hline Control & $4.21 \pm 2.34$ \\
LPS & $19.56 \pm 5.01^{\mathrm{a}}$ \\
LPS + ADAM17-shRNA & $10.35 \pm 3.37^{\mathrm{b}}$ \\
\hline
\end{tabular}

${ }^{\mathrm{a}} \mathrm{P}<0.01$ vs. the control group; ${ }^{\mathrm{b}} \mathrm{P}<0.01$ vs. the LPS group. sTNF- $\alpha$, secreted tumor necrosis factor- $\alpha$; LPS, lipopolysaccharide; ADAM17, ADAM17, disintegrin and metalloproteinase domain-containing protein 17; shRNA, short hairpin RNA.

Table II. mTNF- $\alpha$ expression on macrophages, as detected by flow cytometry (mean \pm standard deviation; $\mathrm{n}=3$ ).

\begin{tabular}{lc}
\hline Group & mTNF- $\alpha$ expression (\%) \\
\hline Control & $2.947 \pm 1.119$ \\
Endotoxemia & $18.743 \pm 3.40^{\mathrm{a}}$ \\
Endotoxemia + lentivirus & $48.15 \pm 1.438^{\mathrm{b}}$ \\
\hline
\end{tabular}

${ }^{\mathrm{a}} \mathrm{P}<0.01$ vs the control group; ${ }^{\mathrm{b}} \mathrm{P}<0.01$ vs. the endotoxemia group. mTNF- $\alpha$, transmembrane tumor necrosis factor- $\alpha$.

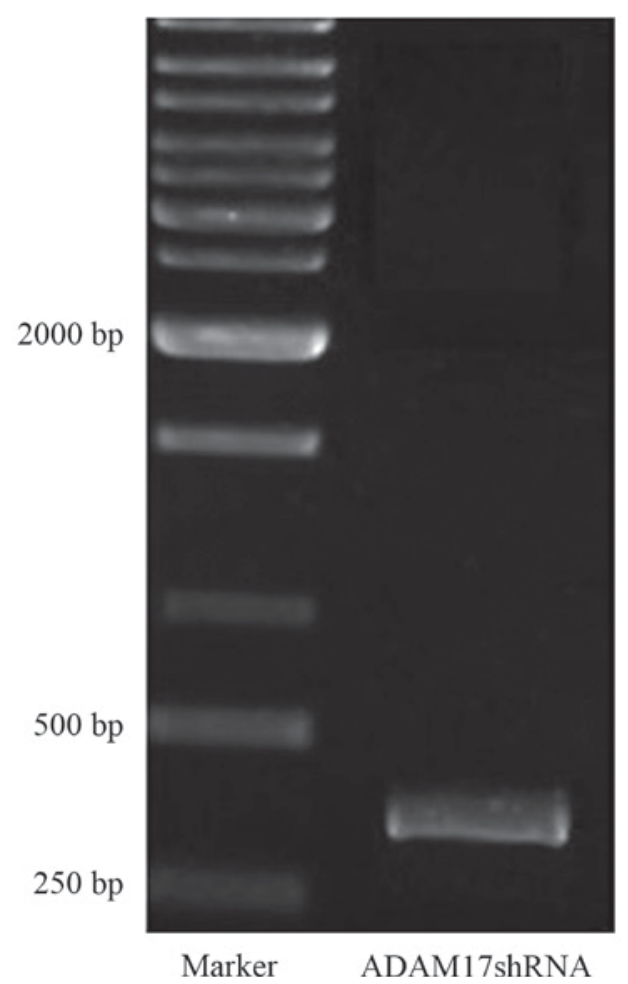

Figure 1. Polymerase chain reaction identification of the lentiviral vector pGC-LV-ADAM17. Representative image exhibiting the expected pGC-LV-ADAM17 band. ADAM17, disintegrin and metalloproteinase domain-containing protein 17

11.0 (SPSS, Inc., Chicago, IL, USA). All data are presented as the mean \pm standard deviation. Where there was homogeneity

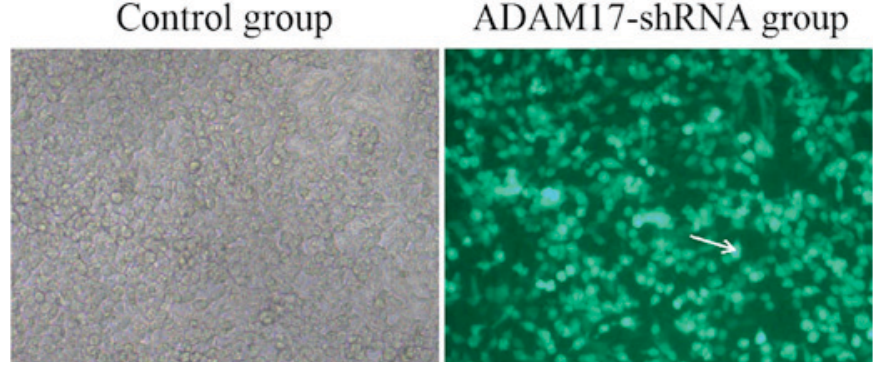

Figure 2. Observation of U937 cells infected with the ADAM17-shRNA lentivirus under a fluorescence microscope (magnification, $\mathrm{x} 200$ ). The green fluorescent protein expression in the U937 cells was detected $72 \mathrm{~h}$ post-infection. The arrow exhibits an example of a stained cell. ADAM17, disintegrin and metalloproteinase domain-containing protein 17; shRNA, short hairpin RNA.
A

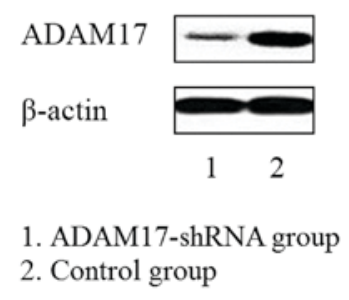

B

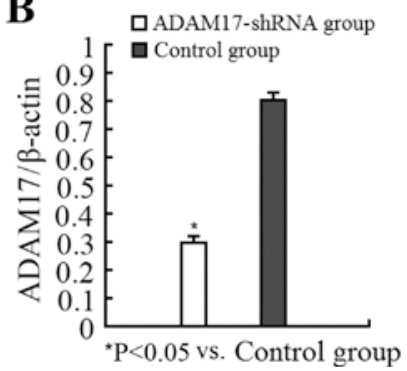

Figure 3. ADAM17 protein expression in U937 cells. (A) Representative image of western blotting demonstrating reduced ADAM17 expression in the ADAM17-shRNA group (B) Ratio of ADAM17/ $\beta$-actin in infected cells. The ADAM17 protein levels in the ADAM17-shRNA group were significantly reduced compared with the control group. " $\mathrm{P}<0.05$ vs. the control group. ADAM17, disintegrin and metalloproteinase domain-containing protein 17 ; shRNA, short hairpin RNA.
A

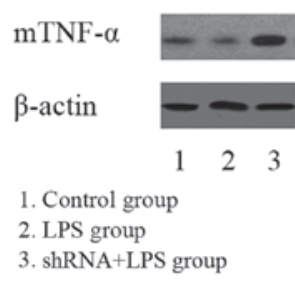

B

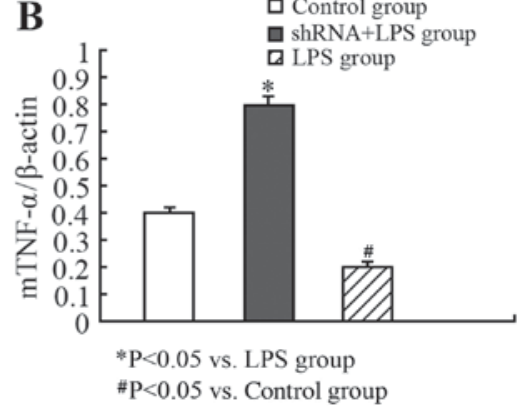

Figure 4. mTNF- $\alpha$ protein expression in the membrane of U937 cells. (A) Representative image of western blotting demonstrating mTNF- $\alpha$ levels following LPS stimulation, in the presence and absence of the ADAM17-shRNA lentivirus. (B) Ratio of mTNF- $\alpha / \beta$-actin in infected cells. The expression of mTNF $\alpha$ on the surface of the U937 cells in the ADAM17-shRNA group was significantly increased compared with the LPS group. ${ }^{*} \mathrm{P}<0.05$ vs. the LPS group; ${ }^{\#} \mathrm{P}<0.05$ vs. control group. mTNF- $\alpha$, transmembrane tumor necrosis factor- $\alpha$; LPS, lipopolysaccharide; ADAM17, disintegrin and metalloproteinase domain-containing protein 17; shRNA, short hairpin RNA.

of variance, a paired Student's t-test was used to evaluate differences between two groups. Otherwise, differences among three groups were determined by one-way analysis of variance with a Student-Newman-Keuls post hoc test. $\mathrm{P}<0.05$ was considered to indicate a statistically significant difference. 

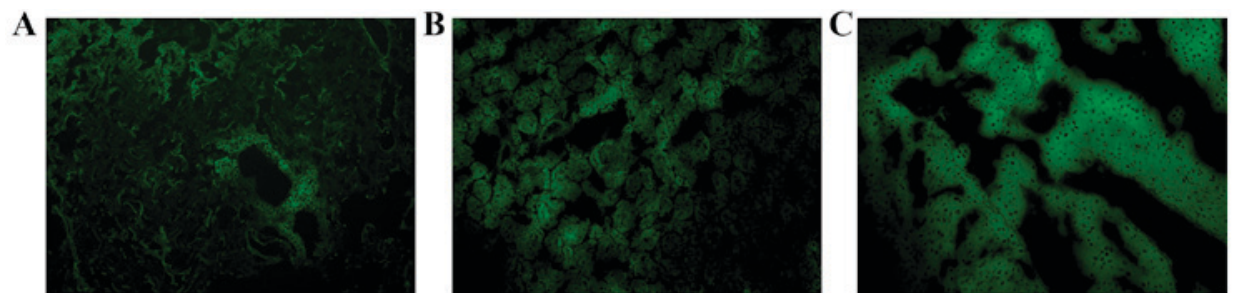

Figure 5. Identification of recombinant lentivirus in mouse tissues following injection. Representative images are exhibited of the (A) lung, (B) kidney and (C) liver. Green fluorescent protein expression indicates the successful introduction of the ADAM17-shRNA lentivirus into mice (magnification, x200). ADAM17, disintegrin and metalloproteinase domain-containing protein 17; shRNA, short hairpin RNA.

\section{Results}

Construction of the ADAM17 shRNA lentiviral vector. In order to construct the ADAM17 shRNA lentivirus (pGC-LV-ADAM17), bacterial clones that had incorporated the target shRNA sequence were identified using PCR analysis; the expected product size was 341 bp (Fig. 1). The recombinant positive clones were sent to Invitrogen (Thermo Fisher Scientific, Inc.) for sequencing, and the nucleotide sequence of the synthetic ADAM17 shRNA was confirmed along with correct insertion into the vector. Subsequently, pGC-LV-ADAM17 was used to transfect $293 \mathrm{~T}$ cells. The viral titer was determined based on GFP expression observed under an inverted microscope. The stock viral titer was $8 \times 10^{8} \mathrm{TU} / \mathrm{ml}$, indicating efficient transfection and successful virus packaging. The infection efficiency in U937 cells was subsequently assessed. A total of $72 \mathrm{~h}$ post-infection, $~ 90 \%$ of the infected U937 cells expressed GFP, indicating that the ADAM17-shRNA lentivirus exhibited a strong tropism for U937 cells (Fig. 2).

Detection of ADAM17 protein using western blotting. In order to determine whether the ADAM17-shRNA lentivirus reduced ADAM17 protein expression, the levels of ADAM17 in infected and control cells were compared. Compared with the control group, the ADAM17 protein levels in infected cells were significantly decreased (Fig. 3) demonstrating that the ADAM17-shRNA lentivirus effectively silenced the ADAM17 gene and inhibited expression at the protein level.

Assessing the effects of the ADAM17-siRNA lentivirus on $L P S$-induced TNF- $\alpha$ secretion in U937 cells. In order to identify the biological function of the ADAM17-siRNA lentivirus, sTNF- $\alpha$ production in response to LPS stimulation in U937 cells was measured using an ELISA (Table I). While LPS stimulation did elicit a TNF- $\alpha$ response in cells infected with the ADAM17-shRNA lentivirus, the concentration of sTNF- $\alpha$ in the ADAM17-shRNA + LPS group was significantly decreased compared with in the LPS group. Since sTNF- $\alpha$ levels are associated with the levels of $\operatorname{mTNF}-\alpha$, the present study additionally assessed whether there were alterations in mTNF- $\alpha$ in LPS-stimulated U937 cells transfected with the ADAM17-shRNA lentivirus. As mTNF- $\alpha$ is a transmembrane protein, western blotting was performed, as described previously (11), to determine the expression of the protein localized to the surface of U937 cells. Compared with the LPS group, there was significantly increased expression of mTNF- $\alpha$ on the surface of the U937 cells in the ADAM17-shRNA + LPS group (Fig. 4), indicating inhibition of TACE activity.
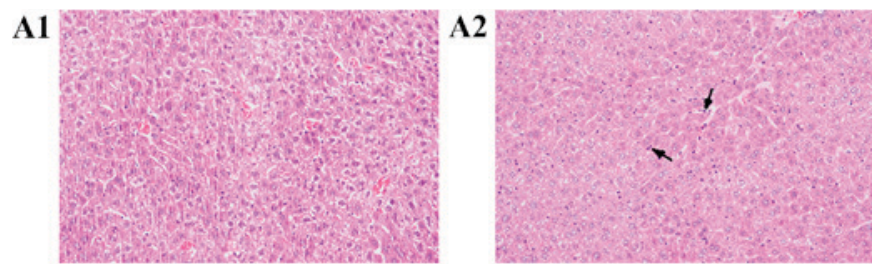

B1
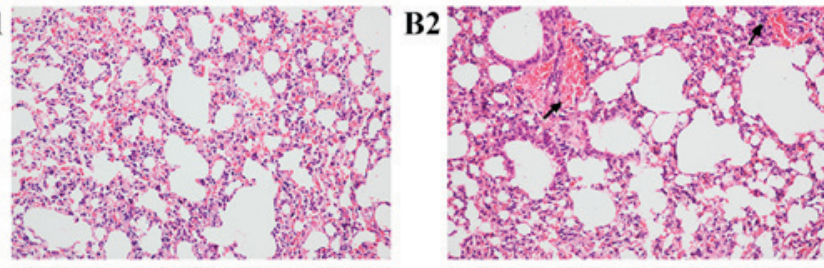

C1

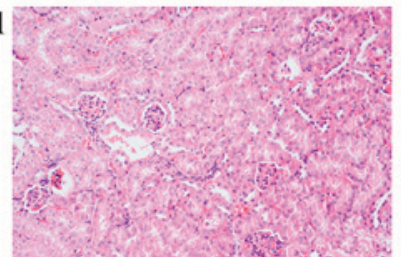

$\mathrm{C} 2$

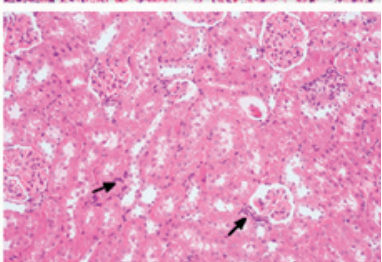

Figure 6. Hematoxylin and eosin staining of the lung, kidney and liver (magnification, x200). Effects of recombinant lentivirus on pathological alterations in the (A) liver, (B) lung and (C) kidney tissues of mice with endotoxemia. Representative images from each group are presented. (A1) Liver slice from a mouse in the endotoxemia + lentivirus group; (A2) liver slice from a mouse in the endotoxemia group; (B1) lung slice from a mouse in the endotoxemia + lentivirus group; (B2) lung slice from a mouse in the endotoxemia group; $(\mathrm{C} 1)$ kidney slice from a mouse in the endotoxemia + lentivirus group; (C2) kidney slice from a mouse in the endotoxemia group. The arrows signify evidence of endotoxemia

Effects of the ADAM17-shRNA lentivirus on a murine model of endotoxemia. In order to determine whether the ability of the ADAM17-shRNA lentivirus to inhibit the production of sTNF- $\alpha$ was physiologically relevant, it was tested in a mouse model of endotoxemia. The lentivirus was injected into the caudal vein of the mice and, upon necropsy, GFP was observed in the liver, lung and kidney, indicating that the recombinant lentivirus was successfully introduced into the mice (Fig. 5).

In response to an LPS challenge, the endotoxemia group exhibited evidence of significant degeneration and necrosis (Fig. 6); this was observed in the liver, kidney and lung tissues $6 \mathrm{~h}$ post-challenge, in addition to a large number of inflammatory cell infiltrates (Fig. 6A2, B2 and C2). Conversely, the mice exposed to the ADAM17-shRNA lentivirus exhibited less LPS-mediated inflammation in the liver, kidney and lung tissues, and fewer signs of degeneration and necrosis. In the liver, hepatic lobule structures were present and there was slight edema in the hepatocytes, in addition to a small amount of inflammatory cell 
A
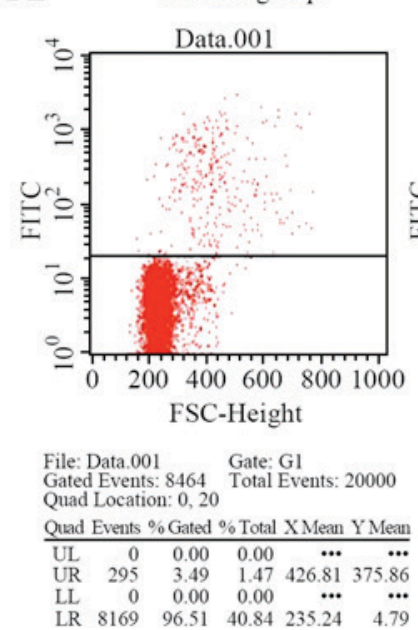

Endotoxemia+ lentivirus group

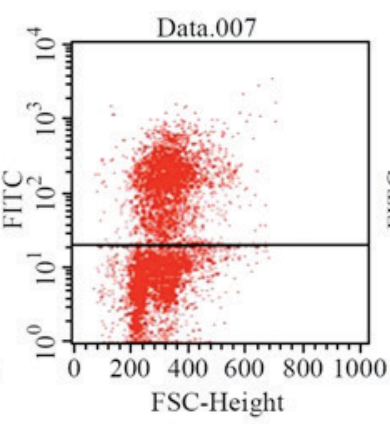

$\begin{array}{ll}\text { File: Data.007 } & \text { Gate: G1 } \\ \text { Gated Events: } 13120 & \text { Total Events: } 20000 \\ \text { Quad Location: 0,20 } & \end{array}$

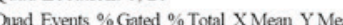

\begin{tabular}{lllllll}
\hline UL & 0 & 0.00 & 0.00 & $\ldots$ & $\ldots$
\end{tabular}

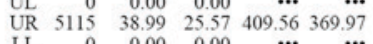

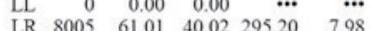

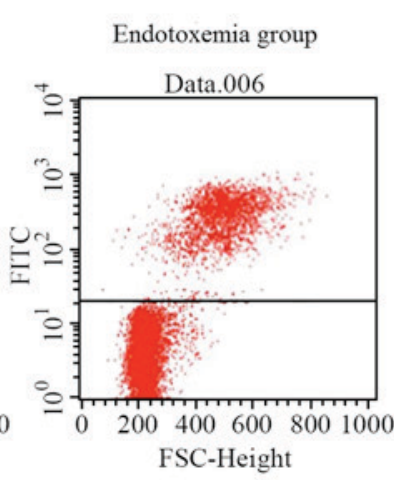

FSC-Height

File: Data.006 Gate: Gl

Gated Events: 8965 Total Events: 20000

Quad Location: 0, 20

Ouad Events \% Gated \% Total XMean YMe

\begin{tabular}{lrrrrr}
\hline UL & 0 & 0.00 & 0.00 & $\cdots$ & $\cdots$ \\
UR & 1857 & 20.71 & 9.28 & 407.43 & 365.95
\end{tabular}

$\begin{array}{llllll}\text { LL } & 0 & 0.00 & 0.00 & \ldots & \ldots\end{array}$
B

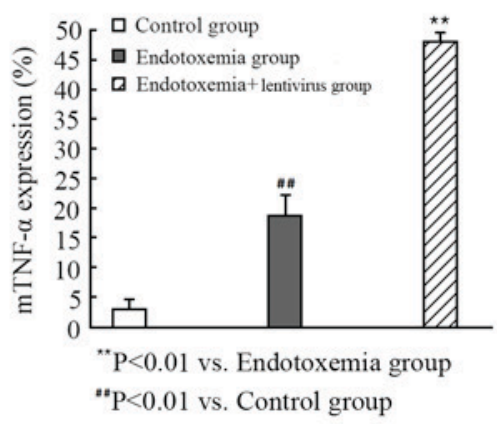

" $\mathrm{P}<0.01$ vs. Control group

Figure 7. mTNF- $\alpha$ expression on macrophages detected by flow cytometry. (A) Representative flow cytometry plots are presented for the control , endotoxemia + lentivirus and endotoxemia groups. (B) Fluorescence intensity of mTNF- $\alpha$ staining in the peritoneal macrophages from mice in the endotoxemia + lentivirus group was increased compared with the endotoxemia group. ${ }^{* *} \mathrm{P}<0.01$ vs. endotoxemia group; ${ }^{\# \#} \mathrm{P}<0.01 \mathrm{vs}$. control group. mTNF- $\alpha$, transmembrane tumor necrosis factor- $\alpha$.

infiltration (Fig. 6A1). In the kidney, the glomerular structure was present and there was slight edema in the proximal tubule epithelium, in addition to mild luminal stenosis (Fig. 6C1). In the lungs, there was a small amount of inflammatory cell infiltration in the bronchi and alveolar septum, although no significant inflammation was observed (Fig. 6B1).

Effects of the ADAM17-siRNA lentivirus on the surface expression of mTNF- $\alpha$ in peritoneal macrophages from mice with endotoxemia. Flow cytometry was used to determine whether exposure to the ADAM17-shRNA lentivirus altered the expression of mTNF- $\alpha$ on peritoneal macrophages following an LPS challenge in mice. The fluorescence intensity of mTNF- $\alpha$ staining in the peritoneal macrophages from the mice exposed to the ADAM17-shRNA lentivirus was increased, compared with the endotoxemia group. This result indicated that the activity of ADAM17 was inhibited, thereby reducing ADAM17-mediated sTNF- $\alpha$ production and increasing the expression of mTNF- $\alpha$ on the cell surface $(\mathrm{P}<0.01$; Fig. 7; Table II).

\section{Discussion}

The present study aimed to determine whether the pathophysiological effects of TNF- $\alpha$, produced in response to an LPS challenge, may be attenuated by regulating the ADAM17-mediated hydrolysis of mTNF- $\alpha$. At present, the only known natural inhibitor of TACE in vivo is metalloproteinase inhibitor 3 (17). The majority of strategies used to reduce sTNF- $\alpha$ have utilized methods to prevent the production of sTNF- $\alpha$ or to elicit enhanced clearance of sTNF- $\alpha$ through vaccinations or antibodies. However, these approaches have led to notable side effects, including inflammation (18). The present study utilized RNA interference (RNAi), which is a relatively novel molecular biology technique. Utilizing lentiviral vectors as a delivery system has several advantages, including: i) Low immunogenicity; ii) the ability to infect cells that are dividing and non-dividing; and iii) the ability to integrate into the host cell genome $(19,20)$. This approach is fundamentally different from the current approaches that utilize anti-sTNF- $\alpha$ antibodies or vaccines. The ADAM17-mediated processing pathway for mTNF- $\alpha$ was selected in the present study as a novel target for inhibiting the overproduction of sTNF- $\alpha$.

In the present study, a lentiviral vector expressing shRNA targeting ADAM17 was constructed, and it was demonstrated in vitro that the lentivirus effectively decreased the protein expression of ADAM17 in U937 cells. The transduction rate exceeded $90 \%$, demonstrating that the lentiviral vector effectively inserted the foreign gene into the host cells. Concurrent with the reduction of ADAM17, levels of sTNF- $\alpha$ in response to an LPS challenge were decreased in cells exposed to the ADAM17-shRNA lentivirus, and protein expression levels of mTNF- $\alpha$ were significantly increased, thus suggesting that the ADAM17-shRNA lentivirus efficiently inhibited the biological activity of ADAM17.

LPS has a strong pyrogenic effect in vivo, and is an important cause of inflammation and various pathological reactions, including SIRS (21). Since the cDNA structure of ADAM17 in mice shares $85 \%$ homology with that in humans, and $91.9 \%$ amino acid similarity, the ADAM17-shRNA lentivirus was tested in a mouse model of endotoxemia. It has previously been observed that lentivirus-mediated RNAi technology is able to achieve high infection efficiency and stable silencing effects in experimental animals (22). Consistent with these findings, the present study observed high levels of GFP expression in the liver, lung and kidney of mice exposed to the ADAM17-shRNA lentivirus. In addition, the mice treated with the ADAM17-shRNA lentivirus had fewer signs of inflammation and less tissue damage in the liver, lungs and kidneys, compared with the control mice, following an LPS challenge. Flow cytometric analysis confirmed that the ADAM17-shRNA lentivirus inhibited the enzymatic cleavage of mTNF- $\alpha$ into sTNF- $\alpha$, resulting in a marked increase in mTNF- $\alpha$ expression in pertioneal macrophages. The results of the present study demonstrated that the ADAM17-shRNA lentivirus may inhibit ADAM-17 mTNF- $\alpha$ cleavage, thereby preventing sTNF- $\alpha$ secretion in response to an LPS challenge. 
In conclusion, the present study successfully constructed a shRNA lentiviral vector targeting the ADAM17 gene, which had obvious in vitro and in vivo effects on TNF- $\alpha$ processing in response to an LPS challenge. The results of the present study may aid the design and improvement of drugs designed to inhibit the function of ADAM17, and suggested a novel means of controlling inflammation and its associated processes.

\section{Acknowledgements}

The present study was supported by the Nature Science Foundation of Hubei Province (grant no. 2012FB04418) and the National Natural Science Foundation of China (grant no. 8100094).

\section{References}

1. Zheng Z, Jiany L, Ye L, Gao Y, Tang L and Zhang M: The accuracy of presepsin for the diagnosis of sepsis from SIRS: A systematic review and meta-analysis. Ann Intensive Care 5: 48, 2015.

2. Ratzinger F, Schuardt M, Eichbichler K, Tsirkinidou I, Bauer M, Haslacher H, Mitteregger D, Binder $M$ and Burgmann H: Utility of sepsis biomarkers and the infection probability score to discriminate sepsis and systemic inflammatory response syndrome in standard care patients. PLoS One 8: e82946, 2013.

3. Kim JH, Seo JW, Mok JH, Kim MH, Cho WH, Kim KU Jeon D, Park HK, Kim YS, Kim HH and Lee MK: Usefulness of plasma procalcitonin to predict severity in elderly patients with community-acquired pneumonia. Tuberc Respir Dis (Seoul) 74: 207-214, 2013.

4. Moss ML, Jin S1, Milla ME, Bickett DM, Burkhart W, Carter HL, Chen WJ, Clay WC, Didsbury JR, Hassler D, et al: Cloning of a disintegrin metalloproteinase that processes precursor tumour-necrosis factor-alpha. Nature 385: 733-736, 1997.

5. Kamisoglu K, Haimovich B, Calvano SE, Coyle SM, Corbett SA, Langley RJ, Kingsmore SF and Androulakis IP: Human metabolic response to systemic inflammation: Assessment of the concordance between experimental endotoxemia and clinical cases of sepsis/SIRS. Crit Care 19: 71, 2015.

6. Rampanelli E, Dessing MC, Claessen N, Teske GJ, Joosten SP, Pals ST, Leemans JC and Florquin S: CD44-deficiency attenuates the immunologic responses to LPS and delays the onset of endotoxic shock-induced renal inflammation and dysfunction. PLoS One 8: e84479, 2013.

7. Yu CW, Juan LI, Hsu SC, Chen CK, Wu CW, Lee CC and Wu JY: Role of procalcitonin in the diagnosis of infective endocarditis: A meta-analysis. Am J Emerg Med 31: 935-941, 2013.

8. Azevedo JR, Torres OJ, Czeczko NG, Tuon FF, Nassif PA and Souza GD: Procalcitonin as a prognostic biomarker of severe sepsis and septic shock. Rev Col Bras Cir 39: 456-461, 2012 (In English, Portuguese).
9. Shu J, He X, Zhang L, Li H, Wang P and Huang X: Human amnion messnchrmal cells inhibit lipopolysaceharide-induced TNF- $\alpha$ and IL-1 $\beta$ production in THP-1 cells. Bio Res 48: 69, 2015.

10. Olofsson PS, Rosas-Ballina M, Levine YA and Tracey KJ: Rethinkinginflammation: Neural circuit $s$ in the regulation of immunity. Immunol Rev 248: 188-204, 2012.

11. Liu Y, Quang P, Braggio E, Badalian-Very G, Flores L, Zhang Y, Sacco A, Maiso P, Azab AK, Azab F, et al: Novel tumor suppressor function of glucocorticoid-induced TNF receptor GITR in multiple myeloma. PLoS One 8: e66982, 2013.

12. Er A and Dik B: The effects of florfenicol on the values of serum tumor necrosis factor- $\alpha$ and other biochemical markers in lipopolysaccharide-induced endotoxemia in brown trout. Mediators Inflamm 2014: 464373, 2014.

13. Locksley RM, Killeen $\mathrm{N}$ and Lenardo MJ: The TNF and TNF receptor superfamilies: Integrating mammalian biology. Cell 104: 487-501, 2001.

14. Lee YJ, Park CH, Yun JW and Lee YS: Predictive comparisons of procalcitonin (PCT) level, arterial ketone body ratio (AKBR), APACHE III score and multiple organ dysfunction score (MODS) in systemic inflammatory response syndrome (SIRS). Yonsei Med J 45: 29-37, 2004.

15. National Research Council (US) Committee for the Update of the Guide for the Care and Use of Laboratory Animals: Guide for the Care and Use of Laboratory Animals, 8th edition. National Academies Press, Washington (DC), pp11-35, 2011.

16. Fan J, Shek PN, Suntres ZE, Li YH, Oreopoulos GD and Rotstein OD: Liposomal antioxidants provide prolonged protection against acute respiratory distress syndrome. Surgery 128 : 332-338, 2000

17. Salem ES, Grobe N and Elased KM: Insulin treatment attenuates renal ADAM17 and ACE2 shedding in diabetic Akita mice. Am J Physiol Renal Pyhsiol 306: F629-F639, 2014.

18. Nicoll JA, Wilkinson D, Holmes C, Steart P, Markham H and Weller RO: Neuropathology of human Alzheimer disease after immunization with amyloid-beta peptide: A case report. Nat Med 9: 448-452, 2003.

19. Katzourakis A, Tristem M, Pybus OG and Gifford RJ: Discovery and analysis of the first endogenous lentivirus. Proc Natl Acad Sci USA 104: 6261-6265, 2007.

20. Chai N, Chang HE, Nicolas E, Gudima S, Chang J and Taylor J: Assembly of hepatitis B virus envelope proteins onto a lentivirus pseudotype that infect sprimary human hepatocytes. J Virol 81: 10897-10904, 2007.

21. Huynh T, Uaesoontrachoon K, Quinn JL, Tatem KS, Heier CR, Van Der Meulen JH, Yu Q, Harris M, Nolan CJ, Haegeman $\mathrm{G}$, et al: Selective modulation through the glucocorticoid receptor ameliorates muscle pathology in mdx mice. J Pathol 231: 223-235, 2013.

22. Hope TJ: VIROLOGY. Visualizing trans-infection. Scinence 350: 511-512, 2015. 